2018-07

Development and validation of Spasticity Index-Amyotrophic Lateral Sclerosis.

\author{
Milinis, $\mathrm{K}$
}

http://hdl.handle.net/10026.1/11413

10.1111/ane.12910

Acta Neurologica Scandinavica

Wiley

All content in PEARL is protected by copyright law. Author manuscripts are made available in accordance with publisher policies. Please cite only the published version using the details provided on the item record or document. In the absence of an open licence (e.g. Creative Commons), permissions for further reuse of content should be sought from the publisher or author. 


\section{Development and validation of Spasticity Index-Amyotrophic Lateral Sclerosis}

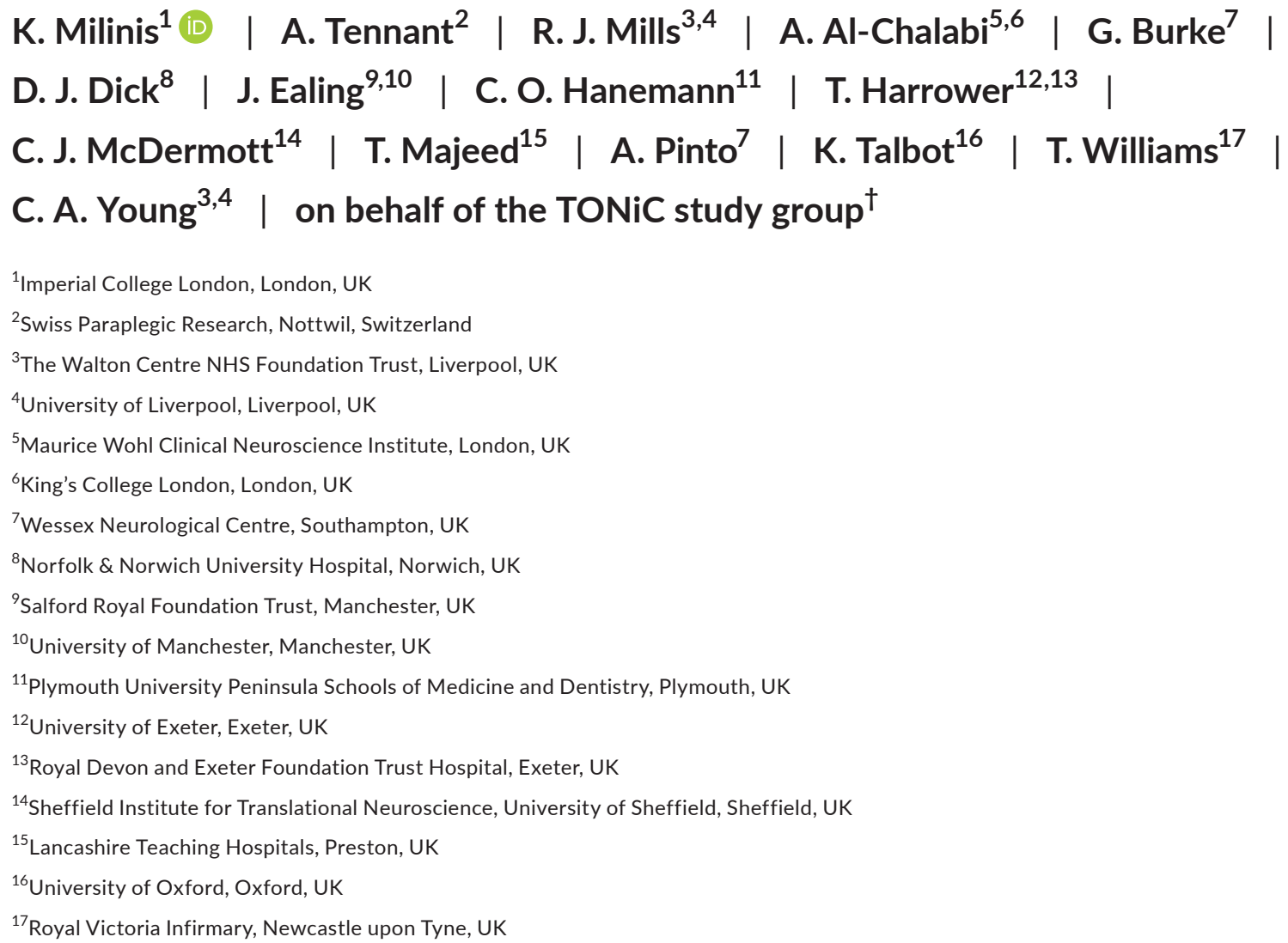

Correspondence

C. A. Young, Walton Centre NHS Foundation Trust, Lower Lane, Fazakerley, Liverpool, UK. Email: carolyn.young@thewaltoncentre. nhs.uk

\section{Funding information}

The TONiC study received support from Biogen, Genzyme, Merck, Motor Neurone Disease Association, NIHR, Novartis, Roche, Teva and Walton Neuroscience Fund. ACC also receives salary support from the National Institute for Health Research (NIHR) Maudsley Biomedical Research Centre.
Objectives: Spasticity is a common and disabling feature of amyotrophic lateral sclerosis (ALS). There are currently no validated ALS-specific measures of spasticity. The aim of this study was to develop and use a self-report outcome measure for spasticity in ALS.

Methods: Following semi-structured interviews with $11 \mathrm{ALS}$ patients, a draft scale was administered across ALS clinics in the UK. Internal validity of the scale was examined using the Rasch model. The numerical rating scale (NRS) for spasticity and Leeds Spasticity scale (LSS) were co-administered. The final scale was used in a path model of spasticity and quality of life. 
Results: A total of 465 patients (mean age 64.7 years (SD 10), 59\% male) with ALS participated. Spasticity was reported by $80 \%$ of subjects. A pool of 71 items representing main themes of physical symptoms, negative impact and modifying factors was subject to an iterative process of item reduction by Rasch analysis resulting in a 20-item scale-the Spasticity Index for ALS (SI-ALS)-which was unidimensional and free from differential item functioning. Moderate correlations were found with LSS and NRS-spasticity. Incorporating the latent estimate of spasticity into a path model, greater spasticity reduced quality of life and motor function; higher motor function was associated with better quality of life.

Conclusions: The SI-ALS is a disease-specific self-report scale, which provides a robust interval-level measure of spasticity in ALS. Spasticity has a substantial impact on quality of life in ALS.

KEYWORDS

amyotrophic lateral sclerosis, motor neurone disease, patient reported outcome measures, quality of life, spasticity

\section{1 | INTRODUCTION}

Spasticity is a key feature in amyotrophic lateral sclerosis/motor neurone disease (ALS/MND) and its subtype primary lateral sclerosis. Patients affected by spasticity experience stiffness, spasms and difficulty with fine motor control. Although a predominant symptom of this disease, there is limited information on the epidemiology, clinical characteristics and treatment of spasticity in ALS. Previous research has shown that spasticity may affect ALS patients in distinct ways compared to other neurological disorders. ${ }^{1}$ For example, people with ALS may experience recurrent bulbar spasticity which is particularly distressing as breathing and speech are interrupted.

The assessment of the effectiveness of spasticity treatments has been a contentious issue, and gold standard measures are lacking. The Ashworth and modified Ashworth scales are the most widely used clinical methods to assess spasticity but have recently been questioned due to concerns over validity, reliability and responsiveness. ${ }^{2,3}$ Studies have shown that findings on examination performed by a clinician do not always correlate with the patient's symptoms regarding severity and location of spasticity, and there is evidence that the Ashworth scale might not represent the patients' experience of spasticity. ${ }^{4,5}$ For example, as the Ashworth scale is a measure of passive muscle resistance, it cannot be used to assess spasticity-related spasms, which can be severe and debilitating for patients. As a result, there has been a growing interest in developing tools that measure spasticity from patients' perspectives across a variety of neurological conditions, to help clinicians understand and adequately treat spasticity and spasticity-related symptoms. Patient reported outcome measures (PROMs) such the Multiple Sclerosis Spasticity Scale-88 (MSSS-88), Patient Reported Impact of Spasticity Measure (PRISM) and Leeds Spasticity Scale (LSS) have been validated in MS, spinal cord injury and stroke, respectively. ${ }^{6-8}$
To date, no self-report measure of spasticity has been developed for patients with ALS. The aim of this study was to develop a robust and reliable patient reported measure of spasticity for ALS.

\section{2 | METHODS}

The study comprised qualitative and questionnaire stages to develop and validate the measure, followed by development of a model of spasticity, disability and quality of life. This work was part of the Trajectories of Outcomes in Neurological Conditions (TONiC) study, which is examining factors influencing quality of life over time in several neurological conditions. All participants received written information, and informed consent was obtained prior to enrolment into the study. Ethical approval was granted from the relevant local research committees (reference 11/NW/0743).

\section{1 | Qualitative stage}

Patients with confirmed ALS were invited to take part in one-to-one, semi-structured interviews at the Walton Centre, Liverpool. All patients were assessed for the presence of spasticity by a neurologist (CAY). A purposeful-sampling technique ${ }^{9}$ of patients with a wide range of spasticity level and distribution, disability, age and disease duration was employed to ensure that the data obtained at the interviews reflected a comprehensive patient experience of spasticity.

A checklist of topic areas was drawn from extant literature on patient experience of spasticity in multiple sclerosis and spinal cord injury. New topics based on the early responses of ALS patients were explored and added to the checklist. The same interviewer (KM) was used throughout. All interviews were recorded and transcribed verbatim. Thematic analysis was carried out during the course of the 
interviews and the list of themes emerged. The interviews were stopped once theme saturation was achieved. A list of questionnaire items retaining original participants' wording was generated to reflect the main themes and subthemes.

A questionnaire containing 71 items was drafted and subjected to a cognitive debriefing with another set of ALS patients in outpatient clinics. Their feedback was used to modify the format, layout and wording of some of the questions. Expert opinion regarding the content of the questionnaire was also sought from the multidisciplinary team members working with ALS patients including neurologists, rehabilitation consultants, physiotherapists and specialist nurses.

A Likert-style response option with four options of "strongly disagree," "disagree," "agree" and "strongly agree" was chosen. There was an introductory question ("Have you experienced stiffness, tightness, spasms or cramps in any muscles including your legs, arms, trunk, neck and throat in the last 2 weeks?") at the beginning of the scale. If participants answered "No," they were instructed to skip the scale.

\subsection{Questionnaire stage}

A questionnaire pack containing the 71-item draft together with other measures was mailed to patients with clinically confirmed ALS recruited at thirteen UK neuroscience centres over a 2-year period. The participants were instructed that the questionnaire should represent their perceptions and experience; those with severe physical disability could have assistance from care givers such as page turning or acting as a scribe. Patients with cognitive impairment or Frontotemporal Dementia (FTD) were not eligible for inclusion. Data from this development sample were subject to Rasch analysis with a view to producing a shortened scale, which was administered to a new, larger validation sample of patients with ALS.

Information on demographic characteristics including age, sex, disease type and duration was also collected. For external validation purposes, the following measures were included in the pack:

1. An 11-point numerical rating scale (NRS) for spasticity with anchors of "no spasticity" and "worst possible spasticity in the last 2 weeks." The NRS has been previously validated in epidemiological studies on spasticity in $\mathrm{MS}^{10}$

2. Leeds Spasticity Scale (SRSS) Scale for Stroke is an 8-item scale, which was developed and validated in patients with stroke-related spasticity. ${ }^{8}$

3. Amyotrophic Lateral Sclerosis-Functional Rating Scale-Revised (ALSFRS-R) is a validated measure of disability in $\mathrm{ALS}^{11}{ }^{11}$ It has been described as multidimensional with a recommendation that it is reported as a profile of bulbar, motor function and respiratory domains, rather than a total score. ${ }^{12}$

4. World Health Organization quality of life assessment (WHOQOLBREF) produces scores for four domains related to quality of life: physical health, psychological, social relationships and environment. ${ }^{13}$
5. Patients were asked to report if they suffer from the loss of emotional control (emotional lability).

Non-responders were contacted by phone after 2-4 weeks. Retest questionnaire was sent to a randomly selected proportion of patients 2 weeks after receiving the first questionnaire. Testretest reliability was examined by differential item functioning (DIF) by time on all who completed the retest, and by concordance correlation coefficient $(C C C)^{14,15}$ on those respondents who indicated that there had been no change in their spasticity. CCC was rated using the Landis and Koch benchmarks: 0-0.20 "slight," 0.21-0.4 "fair," 0.41-0.60 "moderate," 0.61-0.8 "substantial" and above 0.81 "almost perfect" consistency. ${ }^{16}$ Absolute reliability was determined through the Standard Error of Measurement (SEM) and the Smallest Detectable Change (SDC). SEM is calculated as SEM $=$ SD all testing scores $\times \sqrt{ }(1-I C C) .{ }^{17}$ SDC represents the minimal change that a patient must show on the scale to ensure that the observed change is real and not just measurement error and was calculated as $1.96 \times \sqrt{ } 2 \times \mathrm{SEM}$ where 1.96 derives from the $95 \% \mathrm{Cl}^{18}$

\section{3 | Rasch analysis}

The internal validity of the scale was investigated using the Rasch model, a probabilistic measurement model that is now widely used in the health and social sciences. ${ }^{19}$ Detailed description of the process of Rasch analysis can be found elsewhere. ${ }^{20}$ In summary, Rasch analysis is an iterative process, which tests whether the pattern of responses meets the requirements of the Rasch model. In order for the scale to meet model expectations, the following must be satisfied:

1. Fit to the Rasch model (non-significant total chi-square and individual item chi-square statistic)

2. Ordered thresholds

3. Individual item fit residual \pm 2.5

4. Unidimensionality of the scale confirmed if $<5 \%$ of the independent t-tests are found significant

5. Differential item functioning (DIF) absent for age, sex and disability status

6. Reliability (person-item separation index and Cronbach's Alpha $>0.85$ )

7. The absence of local item dependence

\section{4 | Model}

Given fit to the Rasch model, the SI-ALS can be transformed to an interval level metric and could be used in parametric analyses such as path analysis. Thus, a simple illustrative model was postulated whereby the impact of spasticity (SI-ALS) upon quality of life (WHOQOL-BREF Psychological) is partially mediated by motor function (ALSFRS-R Motor). The influences of age, gender and onset type on this model were examined. 


\section{3 | RESULTS}

\section{1 | Qualitative stage}

Eleven patients (seven males) took part in the interview stage. The age range was 40-81 years (median 51) and seven had limb, three bulbar and one respiratory onset of the disease.

The main themes were physical symptoms, negative impact and modifying factors. Physical symptoms were muscle stiffness, spasms and consequences (pain and discomfort). Negative impact was reported on mobility, activities of daily living, social relationships, sleep and mood. Particularly distressing was bulbar spasms, characterized by sudden laryngeal closure and in the most extreme cases causing interference with breathing. None of the patients reported positive effects of spasticity on mobility or transfers.

Among modifying factors, heat, massage, relaxation and antispasticity medication were found to relieve spasticity. Factors that worsened or triggered spasticity were sudden movements, writing, stretching, cold, fatigue and exercise. Bulbar spasms occurred unprovoked or were triggered by yawning, laughing, speaking and eating. Further results of the qualitative study are described elsewhere. ${ }^{1}$

\subsection{Questionnaire stage}

\subsection{1 | Expert panel and cognitive debriefing}

The multidisciplinary panel of clinicians with experience of ALS care endorsed the items as relevant to spasticity. The draft questionnaire was completed face-to-face with a researcher by 17 patients, to ensure the items had face validity and to identify any ambiguities with regard to the instructions, wording and layout.

\subsection{2 | Sample characteristics}

A total of 465 patients returned the questionnaire packs (response rate 51.6\%). The mean age was 64.7 years (SD 10), and 278 (59\%) were male (Table 1). Mean disease duration was 30 months. Limb onset was most common (60\%), followed by Bulbar onset (24.7\%), and $15.3 \%$ had unclassified onset type. There was no difference in onset type across years (chi-square 1.0; $P=.580$ ). The sample was representative of wide range of physical disability with ALSFRS-R Bulbar 0-12, Motor Function 0-24 and Respiratory 1-12. Just over a fifth (22.5\%) reported no spasticity based on NRS-spasticity responses. A total of 178 participants completed the initial 71-item draft scale (development cohort), and a further 287 patients completed the 20 -item reduced item set (validation cohort).

\subsection{3 | Rasch analysis}

Initial fit of the 71-item draft scale to the Rasch model was poor. A substantial breach of the local independence assumption was observed, with residual correlations showing many clusters of variables as close replicas. Each cluster was considered, and the
TABLE 1 Demographic data

\begin{tabular}{|c|c|}
\hline \multicolumn{2}{|l|}{ Characteristics } \\
\hline Total sample & $\mathrm{n}=465$ \\
\hline \multicolumn{2}{|l|}{ Age } \\
\hline Mean (SD) & $64.7(10)$ \\
\hline \multicolumn{2}{|l|}{ Sex } \\
\hline Female (\%) & $187(41)$ \\
\hline Male (\%) & $278(59)$ \\
\hline \multicolumn{2}{|l|}{ Duration of disease (months) } \\
\hline Mean (SD) & $30(40)$ \\
\hline Range & $1-295$ \\
\hline \multicolumn{2}{|l|}{ ALSFRS-R median (range) } \\
\hline Bulbar & $3(0-12)$ \\
\hline Motor & $9(0-24)$ \\
\hline Respiratory & $1(1-12)$ \\
\hline \multicolumn{2}{|l|}{ Employment status } \\
\hline Full-time employment (\%) & $42(9)$ \\
\hline Medically retired (\%) & $109(24)$ \\
\hline Unemployed (\%) & $10(2.2)$ \\
\hline Part-time (\%) & $24(5.2)$ \\
\hline Retired (\%) & $254(54.6)$ \\
\hline Not working for other reasons (\%) & $13(2.8)$ \\
\hline \multicolumn{2}{|l|}{ Questionnaire completion } \\
\hline Independent (\%) & $283(60.9)$ \\
\hline With assistance & $160(34.4)$ \\
\hline Not stated & $22(4.7)$ \\
\hline
\end{tabular}

$\mathrm{SD}$, standard deviation.

item with the best fit or absence of DIF was retained. An iterative phase of item reduction led to 20 items with satisfactory overall fit but some low levels $(<0.2)$ of local response dependency were still observed, relating to symptoms associated in the same region of the body, for example, "My lips go blue when I get spasms in my throat" and "I get spasms in my jaw when I cough." Consequently a testlet solution (10 items +10 items) was employed to minimize any cumulative effect of local response dependency upon fit and dimensionality. Data from the validation stage and the pooled data ( $n=465$ ) had good fit with application of this final solution to the Rasch model (Table 2).

No differential item functioning was observed for age, gender, marital status, onset type or year of data collection (ANOVA $P>.05$ ). Where any spasticity was reported, the person-item distribution was well targeted, with the mean of persons just below the average of the scale (Figure 1).

\subsubsection{Final questionnaire}

All three domains identified at the qualitative stage were represented in the final 20-item Spasticity Index-ALS. 
TAB LE 2 Rasch analysis: SI-ALS development and relevant variables for path model

\begin{tabular}{|c|c|c|c|c|c|c|c|c|}
\hline Scale Stage & \multicolumn{2}{|c|}{ Residual-based fit } & \multicolumn{2}{|c|}{ Chi-square fit } & \multicolumn{2}{|c|}{ Reliability } & \multicolumn{2}{|c|}{ Unidimensionality } \\
\hline $\begin{array}{l}71 \text { item draft } \\
\text { SI-ALS }\end{array}$ & 2.232 & 3.161 & 923.3 & $<.001$ & 0.928 & 0.969 & 25.0 & 22.1-27.9 \\
\hline \multicolumn{9}{|l|}{20 Items SI-ALS } \\
\hline Individual & 1.689 & 1.969 & 167.8 & $<.001$ & 0.892 & 0.891 & 10.8 & 7.2-14.4 \\
\hline Stage 2 testlet & 0.303 & 0.831 & 0.7 & .942 & 0.804 & 0.843 & 4.85 & $1.9-7.8$ \\
\hline Testlet pooled & 0.303 & 0.977 & 10.1 & .432 & 0.820 & 0.862 & 4.95 & $2.6-7.3$ \\
\hline ALSFRS-R-Motor & 0.386 & 0.906 & 15.6 & .210 & 0.67 & 0.76 & 1.1 & $-0.9-3.2$ \\
\hline WHOQoL-Bref Psychological & 3.126 & 0.947 & 8.5 & .748 & 0.79 & 0.75 & 4.4 & $2.4-6.4$ \\
\hline
\end{tabular}

Alpha, Cronbach's Alpha; Cl/LCl, (Lower) Confidence Interval; P, P value; PSI, Person Separation Index; SD, Standard Deviation; Testlet is where items are grouped $10+10$ to remove the effects of local dependency.

FIGURE 1 Person-item distribution

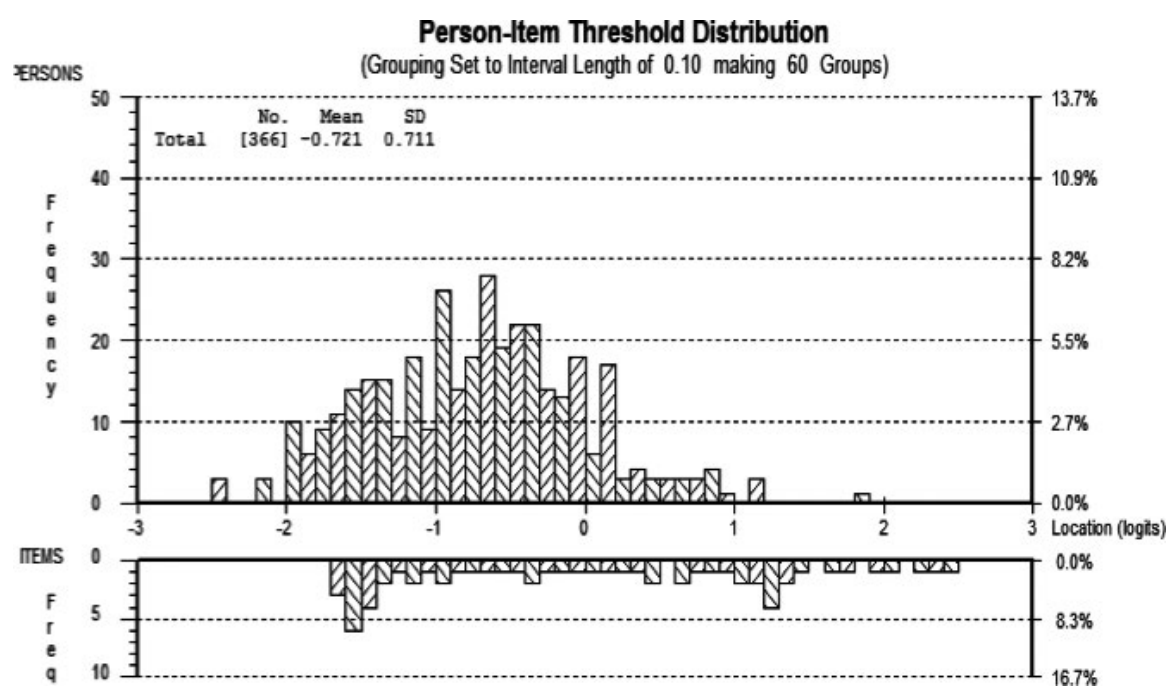

Simple addition of all 20 items to yield a total SI-ALS score (possible range 0-60) yields a valid ordinal score for clinical measurement. If an interval level metric is required for parametric analyses, this total score should be transformed using the transformation (Table S1). Reliability of the scale was 0.87, the Standard Error of Measurement was 2.7 and the Smallest Detectable Change was 7.5. This represents $12.5 \%$ of the total scale width. The correlation between the Leeds Spasticity scale and the SI-ALS was 0.61. Over a quarter (27.5\%) skipped the Leeds Spasticity Scale, indicating no spasticity, whereas $19.6 \%$ skipped the SI-ALS. There was an $80 \%$ agreement in the presence and absence of spasticity between the two scales (defined by their respective skip questions). A significant gradient was also observed across the Numeric Rating Scale with anchors of "No spasticity" and "Worst Possible Spasticity" (F 23.4; $P<.001$ ). About $92 \%$ of participants who reported spasticity on the NRS completed the SI-ALS. Test-retest reliability (Concordance Correlation Coefficient) among those that were stable was substantial at 0.793 (Cl: 0.632-0.889).
A significant association was observed between the level of reported spasticity from the SI-ALS and onset type, with limb onset showing higher levels of spasticity (Kruskal-Wallis chi-square 6.2 (df 2); $P=.046$ ); likewise by age, where those people aged 65 and under displayed higher levels of spasticity (Mann-Whitney U Z; $P<.001$ ). Higher SI-ALS scores were also observed in patients who reported emotional lability (Kruskal-Wallis chi-square 12.62 (df 2); $P=.002$ ).

\subsection{5 | Model}

Variables were entered into the model as Rasch-transformed interval scale estimates. A Sobel-Goodman Mediation Test indicated that $27.7 \%$ of the effect of spasticity upon quality of life was mediated through motor function, supporting a partial mediation. Adding age into the path model for identification purposes, the path model is shown to be an adequate representation of the data (chi-square 0.97 (df1); $P=$.3258) (Figure 2). All paths were significant with the exception of age at $P=.069$, showing a trend where increasing age led to 


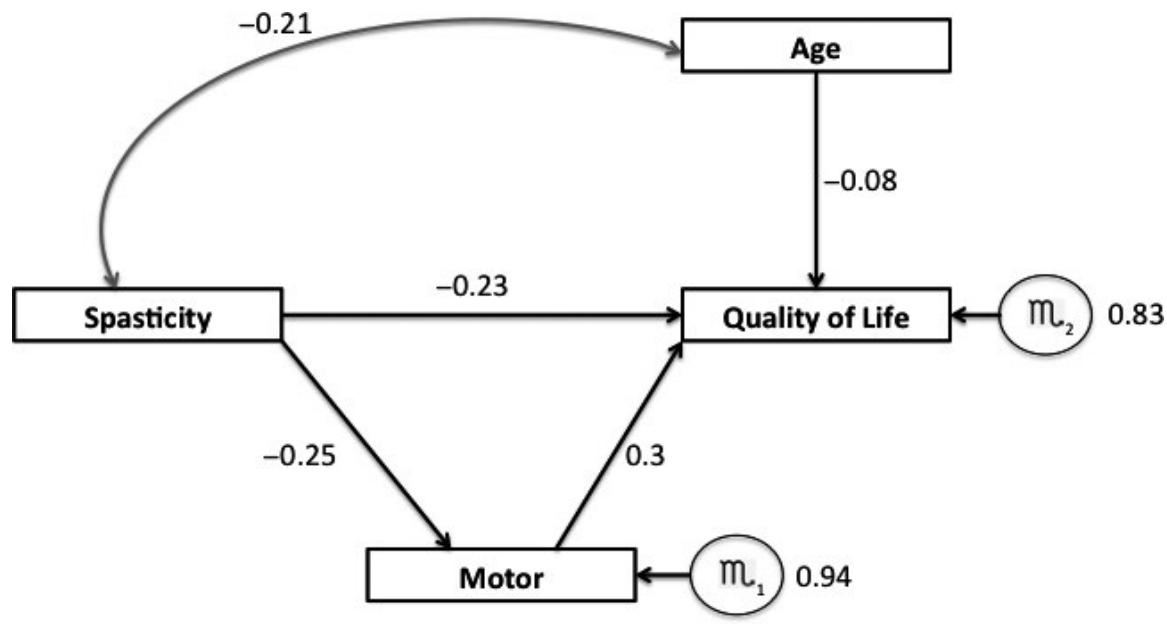

FIGURE 2 The model showing the effects of spasticity, motor function and age on quality of life

worse quality of life. The model confirmed the hypothesized relationships, in that greater spasticity reduced quality of life and motor function, and higher motor function was reflected in a better quality of life. A significant covariance (correlation) was also observed between spasticity and age, with greater spasticity associated with younger age. The total effect of the model showed that the motor scale of the ALSFRS-R dominated the impact upon quality of life.

The model also showed group invariance for onset type across most parameters, with the exception of the relationship between spasticity and motor disability for the unclassified onset group, and the covariance between spasticity and age, which was only significant for the limb onset group (chi-square 4.62 (df 3) $P=.2017$ ).

\section{DISCUSSION}

To authors' knowledge, this is the first study to have investigated ALS patients' experience of spasticity using mixed qualitative and quantitative methods resulting in the development of a patientdriven, interval-level measure of spasticity, which meets the highest standards of psychometric scale development. The SI-ALS comprehensively and accurately reflects patient experience as evaluated in a large cohort with ALS in the UK.

The study demonstrates that spasticity affects the majority of patients with ALS; however, despite its high prevalence there has been a paucity of research on its assessment and treatment. ${ }^{21}$ While spasticity measures have been previously validated in other neurological disorders (stroke, MS, spinal cord injury), such instruments may inadequately capture the experience of spasticity reported by the ALS patients for several reasons. First, the distribution and extent of spasticity differs significantly in ALS compared to other neurological disorders. For example, bulbar spasticity is reported in ALS, while it is rarely seen in other disorders. Secondly, ALS affects both lower and upper motor neurones; therefore, items pertaining to muscle weakness found in other scales could confound the measurement of spasticity in ALS. Finally, items addressing the impact of spasticity are unique to ALS due to the complex relationship between physical symptoms and quality of life in ALS. ${ }^{22}$

\section{1 | Strengths and limitations}

The SI-ALS is a strictly unidimensional measure which gives the advantage of providing a single linear score of spasticity without a need for subscales. The raw scores provide a truly ordinal measure of spasticity, which can be converted to interval level measurement using the transformation table provided (on the proviso that there are no missing data). This facilitates the use of path analysis, as in the above example.

The measure requires the patient, or their scribe, to tick 20 items with a simple four-level Likert scale. Time and ease of administration of the scale is particularly relevant in ALS patients due to high levels of physical disabilities and fatigue. ${ }^{23}$ The scale items reflect patient language and experience, and the cognitive debriefs and validation analysis showed high face validity and patient acceptability. In particular, participants reported satisfaction that bulbar spasticity was included, because for affected patients, bulbar spasticity was a profound symptom and they deplored its omission from generic measures or those developed for other conditions.

The study also has several limitations. Respondents' spasticity was not clinically assessed by a physician and validated against the SI-ALS. This was considered unnecessary and conceptually inappropriate for several reasons. First, the aim of the SI-ALS was to develop a patient-centred outcome measure, which is based on the patient's experience rather than a clinician's observation of spasticity. As a result, the SI-ALS and clinical assessment should be viewed as complementary rather than competing measures of spasticity. Secondly, previous studies have demonstrated that agreement between clinician-administered methods and patient report of spasticity is poor $(r<.5)$ indicating that clinician's assessment is by no means a "gold-standard" measure. ${ }^{4,5}$

\section{2 | Future directions}

The present study serves as a platform for further research into the complex interplay between spasticity, physical and psychosocial factors and quality of life in ALS. Given interval-level data, the SI-ALS provides an opportunity for advanced statistical modelling of quality 
of life determinants, which would further our understanding of the biopsychosocial model of ALS. Further work is needed to determine the minimal clinically important difference of the scale, which could be utilized in clinical trials of anti-spasticity therapies. Finally, external validation of SI-ALS against clinical and biomechanical measures of spasticity would be of interest, as data on patient and clinician agreement on spasticity severity in ALS are lacking.

\section{5 | CONCLUSION}

The SI-ALS is a brief and reliable patient reported outcome measure of spasticity designed for use by people with ALS. Its development is based on ALS patient experience of spasticity and is in line with the Food and Drug Agency (FDA) guidelines for scale development. ${ }^{24}$ Given the scale satisfies the requirements of Rasch measurement, the SI-ALS provides an interval level measurement of spasticity, which is valid for parametric analysis. The scale has high reliability and can be used in all patients with ALS irrespective of age, sex, disease duration and type. It is free for use for academic users and non-profit organizations and can be obtained via contact with the authors.

\section{ACKNOWLEDGEMENTS}

The authors would like to thank all the patients who took part in the study, research and clinical staff for recruitment and the TONiC team.

TONiC study group: David Footitt, Cumbria Partnership NHS Foundation Trust, UK, Email: David.Footitt@cumbria.nhs.uk; Carolyn A. Young, Walton Centre NHS Foundation Trust, Liverpool, UK; University of Liverpool, UK, Email: carolyn.young@thewaltoncentre.nhs.uk.

\section{CONFLICTS OF INTEREST}

Carolyn Young received grants from Motor Neurone Disease Association, NHS Clinical Research Network, Biogen, Novartis, Roche, Teva, Genzyme during the conduct of the study. Christopher McDermott received grants from Orion Pharma, Motor Neurone Disease Association and NIHR outside the submitted work. Ammar Al-Chalabi received grants from Cytokinetics, OrionPharma, Biogen Idec, Chronos Therapeutics, Treeway outside the submitted work.

\section{ORCID}

\section{K. Milinis (iD http://orcid.org/0000-0002-1311-0335}

\section{REFERENCES}

1. Milinis $\mathrm{K}$, Young $\mathrm{C}$. The patient experience of spasticity in motor neurone disease. Amyotroph Lateral Scler Frontotemporal Degener. 2014;15:112-113.
2. Fleuren JF, Voerman GE, Snoek GJ, et al. Perception of lower limb spasticity in patients with spinal cord injury. Spinal Cord. 2009;47:396-400.

3. Pandyan $\mathrm{AD}$, Johnson $\mathrm{GR}$, Price $\mathrm{Cl}$, et al. A review of the properties and limitations of the Ashworth and modified Ashworth Scales as measures of spasticity. Clin Rehabil. 1999;13:373-383.

4. Lechner HE, Frotzler A, Eser P. Relationship between self- and clinically rated spasticity in spinal cord injury. Arch Phys Med Rehabil. 2006;87:15-19.

5. Sköld C. Spasticity in spinal cord injury: self- and clinically rated intrinsic fluctuations and intervention-induced changes. Arch Phys Med Rehabil. 2000;81:144-149.

6. Hobart JC, Riazi A, Thompson AJ, et al. Getting the measure of spasticity in multiple sclerosis: the Multiple Sclerosis Spasticity Scale (MSSS-88). Brain. 2006;129:224-234.

7. Cook KF, Teal CR, Engebretson JC, et al. Development and validation of Patient Reported Impact of Spasticity Measure (PRISM). J Rehabil Res Dev. 2007;44:363-371.

8. Barker S, Horton M, Kent RM, et al. Development of a self-report scale of spasticity. Top Stroke Rehabil. 2013;20:485-492.

9. Bowling A. Research Methods in Health. Buckingham, PA: Open University Press; 2002.

10. Rizzo MA, Hadjimichael OC, Preiningerova J, et al. Prevalence and treatment of spasticity reported by multiple sclerosis patients. Mult Scler. 2004;10:589-595.

11. Cedarbaum JM, Malta E, Fuller C, et al. The ALSFRS-R: a revised ALS functional rating scale that incorporates assessments of respiratory function. J Neurol Sci. 1999;169:13-21.

12. Franchignoni F, Mora G, Giordano A, et al. Evidence of multidimensionality in the ALSFRS-R Scale: a critical appraisal on its measurement properties using Rasch analysis. J Neurol Neurosurg Psychiatry. 2013;84:1340-1345.

13. The WHOQOL Group. Development of the World Health Organization WHOQOL-BREF quality of life assessment. Psychol Med. 1998;28:551-558.

14. Lin LI. A concordance correlation coefficient to evaluate reproducibility. Biometrics. 1989;45:255-268.

15. Lin L-K. Corrections. Biometrics. 2000;56:324-325.

16. Landis JR, Koch GG. The measurement of observer agreement for categorical data. Biometrics. 1977;33:159-174.

17. Chen HM, Hsieh CL, Sing Kai L, et al. The test-retest reliability of 2 mobility performance tests in patients with chronic stroke. Neurorehabil Neural Repair. 2007;21:347-352.

18. Stauffer ME, Taylor SD, Watson DJ, et al. Definition of nonresponse to analgesic treatment of arthritic pain: an analytical literature review of the smallest detectable difference, the minimal detectable change, and the minimal clinically important difference on the pain visual analog scale. Int J Inflamm. 2011;2011:231926.

19. Rasch G. Probabilistic models for some intelligence and attainment tests. Copenhagen: Danish Institute for Educational Research, 1960, and Chicago: University of Chicago Press. 1980

20. Pallant JF, Tennant A. An introduction to the Rasch measurement model: an example using the Hospital Anxiety and Depression Scale (HADS). Br J Clin Psychol. 2007;46:1-18.

21. Ashworth NL, Satkunam LE, Deforge D. Treatment for spasticity in amyotrophic lateral sclerosis/motor neuron disease. Cochrane Database Syst Rev. 2012:CD004156.

22. Gibbons C, Thornton E, Ealing J, et al. The role of fatigue and psychosocial phenomena in the determination of quality of life for patients with motor neurone disease. Amyotroph Lateral Scler. 2013;14:537-545.

23. McElhiney MC, Rabkin JG, Gordon PH, et al. Prevalence of fatigue and depression in ALS patients and change over time. J Neurol Neurosurg Psychiatry. 2009;80:1146-1149. 
24. Arpinelli F, Bamfi F. The FDA guidance for industry on PROs: the point of view of a pharmaceutical company. Health Qual Life Outcomes. 2006;4:85-89.

\section{SUPPORTING INFORMATION}

Additional Supporting Information may be found online in the supporting information tab for this article.
How to cite this article: Milinis K, Tennant A, Mills RJ, et al. Development and validation of Spasticity Index-

Amyotrophic Lateral Sclerosis. Acta Neurol Scand. 2018;00:1-

8. https://doi.org/10.1111/ane.12910 